\title{
Speed Variation during Peak and Off-peak hours on Urban Arterials in Shanghai
}

\section{Xuesong Wangabc*, Tianxiang Fan a, Weinan Lia $^{\text {a }}$, Rongjie Yua ${ }^{\mathrm{a}}$, Darcy Bullock ${ }^{\mathrm{d}}$, Bing Wu ${ }^{\mathrm{a}}$,}

\section{Paul Tremont ${ }^{\mathrm{a}}$}

a School of Transportation Engineering, Tongji University, Shanghai, 201804, China

$\mathrm{b}$ The Key Laboratory of Road and Traffic Engineering, Ministry of Education, China

c Jiangsu Province Collaborative Innovation Center of Modern Urban Traffic Technologies, SiPaiLou \#2, Nanjing 210096, China

d School of Civil Engineering, Purdue University, West Lafayette, IN 47907-2016, USA

* Corresponding author. Tel: +86 21 69583946. E-mail address: wangxs@ tongji.edu.cn.

\begin{abstract}
:
Increased speed variation on urban arterials is associated with reductions in both operational performance and safety. Traffic flow, mean speed, traffic control parameters and geometric design features are known to affect speed variation. An exploratory study of the relationships among these variables could provide a foundation for improving the operational and safety performance of urban arterials, however, such a study has been hampered by problems in measuring speeds. The measurement of speed has traditionally been accomplished using spot speed collection methods such as radar, laser and loop detectors. These methods can cover only limited locations, and consequently are not able to capture speed distributions along an entire network, or even throughout any single road segment. In Shanghai, it is possible to acquire the speed distribution of any roadway segment, over any period of interest, by capturing data from Shanghai's 50,000+ taxis equipped with Global Positional Systems (GPS). These data, hereafter called Floating Car Data, were used to calculate mean speed and speed variation on 234 road segments from eight urban arterials in downtown Shanghai. Hierarchical models with random variables were developed to account for spatial correlations among segments within each arterial and heterogeneities among arterials. Considering that traffic demand changes throughout the day, AM peak, Noon off-peak, and PM peak hours were studied separately. Results showed that increases in number of lanes and number of access points, the presence of bus stops and increases in mean speed were all associated with increased speed variation, and that increases in traffic volume and traffic signal green times were associated with reduced speed variation. These findings can be used by engineers to minimize speed differences during the road network planning stage and continuing through the traffic management phase.
\end{abstract}


Keywords: urban arterial, speed variation, mean speed, influencing variable, Floating Car Data, hierarchical model with random variables 


\section{INTRODUCTION}

Mean speed has been traditionally used as a measure of traffic operational performance, and while it reflects overall time consumed during a trip, it does not provide sufficient information to evaluate the conditions leading to changes in operational performance. Speed variation reflects speed differences among vehicles and speed changes of any single vehicle, and can provide key additional information on operational conditions.

Excessive speed variation can undermine the stability of traffic flow (Verhoef et al., 1999). They found that speed differences were one of the most important reasons for congestion, especially outside peak hours. The situation where high speed vehicles change lanes to pass low speed vehicles often slows vehicles on adjacent lanes, and is one example of how speed differences can lead to congestion.

Increased speed variation can also affect safety (Solomon, 1964; Cirillo, 1968). These researchers found that crash counts were the lowest for vehicles travelling near the mean speed, and increased as drivers moved away from the mean speed. Greater speed variation has also been shown to lead to greater headway differences, and consequent serious injuries (Aarts and Schagen, 2006; Wang et al., 2015).

Previous research has shown that traffic flow, mean speed, traffic control parameters and geometric design features may influence speed variation (Figueroa and Tarko, 2005; Dinh and Kubota, 2012). However, relationships observed among these variables have differed in these studies. One reason may be that they all acquired speed data at specific locations, rather than throughout the roadway segments under study. Such spot speed collection methods are not adequate for acquiring distributions of speeds along roadway segments because the speeds of vehicles can change at any location along a segment. Global Positioning Systems (GPS) can provide a location and time stamp for every equipped vehicle, and this allows speed data acquisition to reach a new stage where speeds can be obtained at any point or throughout the segment. This speed data acquisition method is hereafter called the Floating Car Data (FCD) method. Shanghai has a 50,000+ vehicle taxi fleet equipped with GPS, and capturing data from this fleet makes it possible to adopt the FCD method to measure speeds wherever desired throughout its $10,000 \mathrm{~km}+$ road network.

The current study used the FCD method by recording sequentially the location of each taxi in the sample approximately every ten seconds along the selected road segments. This position and time data was then used to calculate mean speeds and speed variations along the selected segments. Locations along road segments where taxis were recorded had an equal probability of being selected, and this ensured full coverage of the segment. The FCD method also allowed any individual vehicle to be tracked, enabling examination of speed differences among vehicles and speed changes of any single vehicle throughout the sampled segments.

The objective of this study was to identify factors associated with speed variation that may later be used to develop guidelines for improving operational performance. Based on 234 road segments from 8 urban arterials, traffic flow, mean speed, traffic control parameters and geometric 
design features were acquired to estimate their role as potential influencing variables. Mean speed and speed variation were calculated using FCD. Bayesian hierarchical models with random variables were developed to account for spatial correlations among segments within each arterial and to accommodate heterogeneities among different arterials. Considering that operational conditions vary over the day, AM peak, Noon off-peak, and PM peak hours were examined separately.

\section{LITERATURE REVIEW}

\subsection{Spot and FCD Speed Collection Methods}

Researchers have mainly adopted two collection methods to measure speeds, spot methods and the Floating Car Data (FCD) method. Spot methods commonly use loops, radar or laser to collect speeds at specific points along a roadway segment, while the FCD method uses GPS equipped vehicles to acquire speeds along complete roadway segments. Poe et al. (1998) used loop detectors to collect speeds and compared speed characteristics at different cross-sections. They found the speed at the middle point of a road segment was the highest. This finding shows that the selection of measuring points determines the speeds acquired and that such speeds may not be representative of speeds over the entire segment. Another shortcoming of spot methods is their inability to capture speed changes of single vehicles along road segments. In contrast, by recording locations and times of each floating car, the FCD method is better for acquiring mean speed and speed variation data along a full segment. Readers can refer to Xie et al. (2013) and Wang et al. (2015) for more details.

\subsection{Selection of a Modeling Approach}

Modeling approaches depend on the data distributions and structures, the scales of measurement, and the purpose and design of the study. When response variables are continuous and normally distributed, simple linear models are most suitable. Within this category of models, Multiple Linear Regression (MLR) models are frequently used. Wang et al. (2014) used MLR models and found associations between geometric design features and mean speed on urban arterials. However, these simple linear models fail to accommodate dependent data structures often seen in these kinds of studies. Hierarchical models offer one approach to handling the lack of independence problem by separating samples into different levels and assigning each level a random effect term to account for correlations among samples. For example, an arterial-level random effect term that represents a common unknown variable for all segments within an arterial can correct for spatial correlations among segments. Using a two-level hierarchical model, Park and Frank (2006) found increases in the radius of curves were associated with decreases in speed variability. Hierarchical models are also well suited to accommodating data that falls on different measurement scales. Wang et al. (2015) included both ratio-scale variables (e.g. number of lanes) and nominal-scale variables (e.g. median types) in Bayesian hierarchical models. Depending on the study purpose, there are different types of designs used, although the most common types are 
the before-after and cross-sectional. The before-after approach is better at identifying effects of certain pre-identified variables, while the cross-sectional approach is better at determining the role of potential variables when not enough information is available to identify the specific variables of interest. Other issues enter into model selection, however, a full consideration of these is beyond the scope of this paper.

\subsection{Models for Speed and Speed Variation}

Given that speed variation was normally distributed, and that speed variations between consecutive road segments were not independent, a modeling approach compatible with these characteristics is needed. Previous studies used hierarchical models in these situations, but they still had the shortcoming of not fully considering impacts of unmeasured characteristics on dependent variables (Karlaftis and Tarko, 1998). Such heterogeneities, as they are commonly called, can lead to changes in the effects of influencing variables across groups (i.e., in this study, segments within an arterial). For example, although not easily measured, differing road surface conditions on arterials may be associated with speed variation. We addressed this heterogeneity problem by separating the data structure into arterial and segment levels and by treating influencing variables as random variables (specifying coefficients of these variables for each arterial). Therefore, hierarchical models with random variables, a type of cross-sectional approach, were used in this study.

\section{DATA PREPARATION}

This section will describe the road segment sample selection criteria, variables and data acquisition methods.

\subsection{Road Segment Sample Selection}

Road segments selected were in a single direction only and satisfied the following criteria:

(1) Shanghai downtown location.

(2) surface roads (tunnels and elevated segments were excluded).

(3) loop detectors were functional.

Table 1 summarizes the key features of the selected arterials.

\section{Place Table 1 about here}

\subsection{Segment Data Collection}

Variables were separated into arterial level and segment level. Three categories of variables were collected at the segment level: geometric design features, traffic volume, and traffic control parameters. Attributes and summary statistics of these variables are presented in Table 2. Geometric design features were obtained using a Geographic Information System (GIS) map of Shanghai and supplemented with data acquired in field investigations. Traffic volume was obtained using loop detectors during AM peak hours (7:00-9:00 a.m.), Noon off-peak hours (12:00-2:00 
p.m.), and PM peak hours (4:30-6:30 p.m.). One concern was that correlations among independent variables would lead to errors in estimates. This problem, commonly referred to as multicollinearity, was avoided by using average traffic volume, an aggregate measure not closely correlated with any of the independent variables. Because the focus was on speed variation along segments, speed variation needed to be measured independently of vehicle speed changes on turn lanes, and therefore only speeds on through lanes were measured. Traffic control parameters were acquired from the Sydney Coordinated Adaptive Traffic System (SCATS). To improve the reliability of estimates, a multicollinearity test was conducted in SAS ${ }^{\circledR}$ (SAS Institute Inc., 2002). If the Variance Inflation Factor (VIF) value of any independent variable is greater than ten, that variable should be removed (Cody and Smith, 2011). Therefore, not all the variables shown in Table 2 were included in the final models.

Variables of curvature, grade, lane width, and speed limits were excluded from the models because they did not change much along the sample arterial segments. That is, most of the selected road segments were fairly straight, grades of segments were minimal, arterial lane widths were mostly fixed at $3.5 \mathrm{~m}$, and posted speed limits signs were not present.

\section{Place Table 2 about here}

\subsection{Quality of Floating Car Data from Taxis}

Heavy traffic volume conditions in downtown Shanghai limit speed differences between taxis and other vehicles. It has been shown that taxis without carrying passengers travel more slowly than the general traffic stream (Liu et al, 2009), so these were excluded from the sample. To ensure taxi speeds were representative of overall speeds, the sample size and sampling interval both met the minimum requirements of estimating speeds with the FCD method. Readers can refer to an earlier study in which Wang et al. (2015) presented a detailed discussion on the representativeness of taxis including sample size and sampling interval.

\subsection{Speed Data Acquisition}

Floating car data were sampled on three consecutive Wednesdays in May, 2011 during the AM peak, Noon off-peak and PM peak hours. Each sample reached 5-8\% of total arterial traffic volume, a size adequate for a 95\% confidence level in speed estimates (Chen and Chien, 2000; Cheu et al., 2002). If the sampling interval exceeded 30 seconds, the data were excluded. However, more than $85 \%$ of the tracked vehicles were sampled at ten second intervals, a level that met the accuracy requirements of the study.

Three steps are needed to acquire speed data throughout a segment using the FCD method. First, extract vehicle ID, time stamps and coordinates as a series of GPS records from sampled taxis. Second, geocode those GPS records to the road to obtain the actual path traversed by each tracked vehicle. Third, compute the average running speed of each vehicle through the segment. More details can be found in an earlier study by Wang et al. (2015). 
Figure 1 illustrates a speed profile for a sample vehicle. The $\mathrm{x}$-axis represents distance to a start point of a segment, and the y-axis represents average running speed between two GPS records. The red points represent the positions of the sampled taxi on segments after map matching.

\section{Place Figure 1 about here}

Figure 2 shows speed profiles of two selected arterials during AM peak hours. Each curve represents an individual vehicle. On arterial 1, vehicles stop less frequently than on arterial 2 . The blue line highlights one vehicle's speed profile. It shows that a vehicle may reach its maximum speed at locations that differ from others along a segment, a finding consistent with Poe et al.'s (1998) observation.

\section{Place Figure 2 about here}

\subsection{Mean Speed and Speed Variation Calculations}

The mean speed and speed variation of a segment were calculated using all the sample vehicles that traveled on the segment during the time period of interest. On occasions when taxis operated at speeds less than $5 \mathrm{~km} / \mathrm{h}$, operational conditions at the segment level could not be represented because those low speed data were mostly influenced by a red light signal at intersections and by passengers' entering and exiting taxis. Consequently, average running speeds between consecutive GPS records below $5 \mathrm{~km} / \mathrm{h}$ were excluded.

The mean speed of a segment was calculated by obtaining each single vehicle's speed over the time period of interest, and then averaging those speeds. To consider a specific example, if vehicle $\mathrm{j}$ left $\mathrm{N}$ records during its trip through the segment, its single vehicle speed was computed by as follows.

$$
V_{j}=S /\left(T_{j}-D_{j}\right)
$$

where $V_{j}$ is the speed of vehicle $\mathrm{j}$ on a segment; $\mathrm{S}$ is the length of the segment; $T_{j}$ is the travel time vehicle $\mathrm{j}$ spent on this segment; $D_{j}$ is the low-speed time delay of vehicle $\mathrm{j}$ on this segment where the average speed between two consecutive GPS records is below $5 \mathrm{~km} / \mathrm{h}$.

Then, the mean speed of a segment was computed as follows.

$$
\bar{V}=\sum_{j=1}^{n} V_{j} / M
$$

where $V_{j}$ is the single speed of jth vehicle travelled through the segment during AM peak, Noon off-peak or PM peak period; $M$ is the number of vehicles on the segment during that period.

The speed variation of a segment was calculated by acquiring each tracked vehicle's average running speed (i.e. speeds between consecutive GPS records), and then taking a sample standard deviation of those running speeds. It was calculated as follows:

$$
D_{v}=\sqrt{\sum_{i=1}^{N-M}\left(v_{i}-\bar{v}\right)^{2} /(N-M-1)}
$$

where $v_{i}$ is the average running speed between the ith position and the (i+1)th position from GPS records on the segment during AM peak, Noon off-peak or PM peak period; $\bar{v}$ is the mean value of all $v_{i}$; $\mathrm{N}$ is the number of GPS records; and $\mathrm{M}$ is the number of vehicles on the segment during 
the time period of interest.

\section{PRELIMINARY ANALYSIS}

Table 3 lists mean speeds and speed variations during the different time periods for the eight arterials.

\section{Place Table 3 about here}

On most of these arterials, both mean speeds and speed variations in one direction are lower than those in the other direction during the AM peak periods but higher than those in the other direction during the PM peak periods, reflecting commuting patterns. Arterial 5 data presented in Figure 3 is an example that shows this. We can see that speed variations of an arterial differ across time periods, and this is the reason we developed separate models for the different time periods.

\section{Place Figure 3 about here}

Before quantification of the relationships between mean speed and speed variation using statistical models, a general sense of how the relationship changes over peak and non-peak periods can be seen by viewing Figure 4. In the figure, sequences of segments plotted on the x-axis are ranked by mean speeds. It can be seen that during peak periods speed variations tend to increase with increases in mean speeds. However, speed variation fluctuates substantially during each time period, and this indicates that there are other potential variables influencing speed variation.

\section{Place Figure 4 about here}

\section{MODELING APPROACH}

\subsection{Decision Tree Models}

Decision tree models allow variables to be classified by segmenting the data set into small homogenous groups (Elmitiny, 2010). This segmentation helps to reveal nonlinear relationships among variables (Chang and Wang, 2006). In the current study, decision tree models were developed for both mean speed and traffic volume using SPSS ${ }^{\circledR}$ software (IBM Corp, 2010). To ensure the best partitioning arrangement, a measure was calculated using the principle of entropy. As an example, if segment samples $\mathrm{S}$ into categories $\mathrm{C}$, for which the proportion of examples in $\mathrm{C}_{\mathrm{i}}$ is $\mathrm{P}_{\mathrm{i}}$, then the entropy of $\mathrm{S}$ is:

$$
\operatorname{Entropy}(S)=\sum_{i=1}^{n}-p_{i} \log _{2}\left(p_{i}\right)
$$

The smaller the entropy, the better the partitioning is. Table 4 shows the grouping results.

\section{Place Table 4 about here}

\subsection{Hierarchical Models}

It was mentioned earlier that road segments within an arterial share correlations attributable 
to similarities in geometric design features. These correlations may influence predictions of crash occurrence. Different arterials have some unobserved characteristics associated with speed variation, and these so-called heterogeneities may cause variables to have different effects across arterials. Hierarchical models offer a promising way to handle both the correlation and heterogeneity problems that exist in data structures (Cruzado and Donnell, 2010; Park and Frank, 2006). However, the hierarchical models used in most of prior studies introduced a random effect term to represent heterogeneities, and this did not reflect possible changes of variable effects. In this study, a random variable modeling approach was adopted, and this allowed coefficients of variables to differ for different arterials.

The hierarchical models with random variables were expressed as:

$$
\begin{gathered}
y_{i} \sim N\left(X_{i}^{0} \beta^{0}+X_{i} B_{j[i]}, \sigma_{y}^{2}\right), \text { for } i=1, \cdots, n \\
B_{j} \sim N\left(U_{j} G, \sum B\right), \text { for } j=1, \cdots, J
\end{gathered}
$$

where $X_{i}^{0}$ is a matrix of predictors whose coefficients do not vary by groups and $\beta^{0}$ is the vector of their regression coefficients. Model (5) is sometimes called mixed-effects regression, where the $\beta^{0}$ 's are the fixed parameters and the B's are the random parameters. $B$ is the $J \times K$ matrix of individual-level coefficients; $U$ is the $J \times L$ matrix of group-level predictors (including the constant term). $G$ is the $L \times K$ matrix of coefficients for the group j, so $U_{j} G$ is a vector of length $K$. $J$ is the number of divided groups (16 groups in this study); $K$ is the number of individual-level predictors (including the intercepts) that vary by group; $L$ is number of group-level predictors (including the intercepts). The variance-covariance matrix $\sum B$ is set to follow the inverse-Wishart distribution with the unscaled covariance matrix $Q$,

$$
\begin{aligned}
& \sum B=\operatorname{Diag}(\varepsilon) Q \operatorname{Diag}(\varepsilon) \\
& \mathrm{Q} \sim \operatorname{Inv}-\text { Wishart }_{K+1}(I)
\end{aligned}
$$

and which has computational convenience (Gelman and Hill, 2007). The variances $\sigma_{k}^{2}$ can be achieved as

And the covariance $\sum_{k l}$ is

$$
\sigma_{k}^{2}=\varepsilon^{2} Q_{k k}, \text { for } k=1, \cdots, K
$$

$$
\sum_{k l}=Q_{k l} \varepsilon_{k} \varepsilon_{l}, \text { for } \mathrm{k}, \mathrm{l}=1, \cdots, \mathrm{K}
$$

\subsection{Bayesian Estimation}

The model results were estimated using the Bayesian approach. This approach, in contrast to the classical statistical approach, considers prevailing conditions (prior distributions of variables) before collecting sample data, and then, following Bayes' theorem (Bayes and Price, 1763) calculates posterior distributions using both the priors and the sample. The key idea of the Bayesian approach is that it allows modification of current probability calculations based on information 
from previous probability calculations. This can avoid biased estimation attributable to a small sample size. The prior distributions can be of two types, uninformative and informative. Informative priors often come from previous experiments or expert opinions. If such data are not available, uninformative priors are suggested. The most commonly used uninformative priors are normal distributions with a mean of zero and a variance of 10,000, and these were adopted in this study. To obtain posterior distributions, Markov Chains consisting of the uninformative priors and observed samples were generated and iterated until convergence. Next, based on Monte Carlo algorithm, sufficient random numbers from these posterior distributions were used to calculate posterior summaries (e.g. means and standard deviations). These two steps, usually called the simulation-based Markov Chain Monte Carlo (MCMC), were accomplished via WinBUGS (Imperial College \& MRC, 1996-2003) software. The Deviance Information Criterion (DIC) was used to measure model accuracy and complexity (Spiegelhalter et al., 2003). Smaller DIC values indicate a better goodness-of-fit. If two models' differences in DIC are larger than ten, the higher DIC model is ruled out. The Bayesian Credible Interval (BCI) was used to judge if a variable was significant (Gelman et al., 2003). For instance, a variable is significant if its BCI did not contain a value of zero.

\section{MODELING RESULTS}

Considering random variables and possible nonlinear relationships, four different models for each time period were developed as follows:

(1) Hierarchical models with fixed parameters, using random effects to account for unobserved heterogeneity between different arterials.

(2) Hierarchical models with fixed parameters, considering nonlinear relationships for mean speed and volume.

(3) Hierarchical models with random variables, treating speed and traffic volume as random variables.

(4) Hierarchical models with random variables, treating mean speed as a random variable and considering a nonlinear relationship for volume.

Correlation coefficients of the influencing variables were calculated to test for multicollinearity, to ensure the variables entered into the models would not cause incorrect estimates. Table 5 shows goodness-of-fit of these four models.

\section{Place Table 5 about here}

For the three different time periods, the DIC values showed the model that treated speed as a random variable and the relationship between speed and volume as nonlinear provided the best fit. This type of model was used to examine the variables that influence speed variation. The results are shown in Table 6. 


\section{Place Table 6 about here}

6.1 Variables that increase speed variation

Higher mean speed was associated with greater speed variation in all the models. This can be seen in the Preliminary Analysis section. Compared to the AM peak and the PM peak models, the coefficient of mean speed in the Noon off-peak model was the smallest. The standard deviation of mean speed coefficient was 0.020 in the off-peak model. This was lower than the other two models ( 0.039 for AM peak periods and 0.026 for the PM peak periods). A reason could be that traffic volume differed much more during peak than off-peak periods among arterials.

Number of lanes (a categorical variable) was positively related to speed variation in all the models. This is consistent with Cruzado and Donnell's (2010) finding, and may occur because vehicles have more space to change speeds on arterials with more lanes.

Number of access points held a positive sign in all the models, indicating speed variation would increase as access points increased. This likely occurs because arterial traffic is more frequently interrupted as traffic from other roadways enters the stream.

Bus stops, another categorical variable, was positively associated with speed variation in both AM peak and PM peak models, but not during Noon off-peak periods. One possible explanation was that stopping buses would slow down the traffic at adjacent lanes, and the fewer buses (and fewer other vehicles) during the off-peak periods, left enough space for neighboring vehicles to overtake stopping buses.

\subsection{Variables that reduce speed variation}

Higher traffic volume was associated with reduced speed variation in both AM peak and PM peak models. One possible explanation is that heavy traffic during peak periods restricts vehicle speeds. However, in the Noon off-peak model, traffic volume did not show a significant association with speed variation. A possible reason could be that during off-peak periods, there is more space for vehicles to choose driving speeds, and this leads to fewer interactions among vehicles.

Green time held a negative sign in all the models, meaning speed variation decreased as green time increased. As expected, fewer stops led to less variation.

\section{SUMMARY}

The objective of this study was to examine relationships between influencing variables and speed variation using Floating Car Data to measure mean speeds and speed variations across entire roadway segments.

A preliminary analysis of speed variation was conducted prior to selecting modeling strategies. Based on speed profiles and diagrams of mean speed against speed variation, three characteristics were discovered as follows:

(1) speeds of sample vehicles differed on the same segments and during the same time period; 
(2) speed variation changed across time periods on the same segments;

(3) large differences in speed variation occurred on segments with similar mean speeds during the same time period.

These three characteristics show that heterogeneities exist among arterials, and that changes in traffic volume throughout the day should be considered. Therefore, hierarchical models that treat mean speed as a random variable were developed for AM and PM peak periods, and Noon off-peak periods separately.

Modeling results showed increases of mean speed and number of lanes and access points, and presence of bus stops were associated with increases of speed variation, while increases of traffic volume and green time were accompanied by decreases of speed variation.

\section{GENERAL DISCUSSION}

The models showed that speed variation increased as mean speed increased. This is in contrast to Figueroa and Tarko's (2005) finding that a decrease of mean speed was associated with an increase of speed variation. Several reasons could be offered to explain these contradictory findings. Their study was based on two-lane highways with speed limits, while our study focused on urban arterials with two to five lanes and without posted speed limit signs. When there were only two lanes, high mean speeds tell us that most vehicles on a segment all traveled at high speeds. However, when there were more than two lanes, vehicles on outer lanes (close to the shoulder lane) could travel at low speeds even if the mean speed of a segment was high. Also, considering that there were no intersections on highways, vehicles on the outer lanes could travel at higher speeds on the two-lane highways than on urban arterials. Another reason for the different findings could be that speeding is not a chargeable offense on Shanghai arterials, and drivers will choose to drive faster when there is an opportunity.

As the FCD method can provide more accurate speed data than spot speed collection methods, important improvements can be made in several fields of transportation. Seo and Kusakabe (2015) used probe vehicle data (i.e. floating car data) to develop a traffic state estimation method. Pascale et al. (2015) compared floating car data with previously identified historical speed patterns to detect anomalous events. In this study, the findings can be used to develop guidelines for authorities to reduce speed variation on urban arterials, and thus improve operation and safety performance. Poor operational performance (especially congestion) is severe in Shanghai, and various strategies are needed to reduce it. At the planning stage of a road network, the number of access points is a major design decision that affects capacity, and consequently congestion. Reducing access points will reduce speed variation and lead to reduced congestion. Other major considerations when designing an urban arterial are the number of lanes and the frequency of bus stops. Adding lanes is a strategy to improve road capacity. However, more lanes can lead to greater speed variation and thus rendering the benefits less than anticipated. Development of public transport is at fast pace in Shanghai, and more bus lines will necessarily be accompanied by more bus stops, leading to increased speed variation. In the stage of traffic management, speed limits 
and traffic signal control are key strategies to smooth traffic speeds. However, given that we found increases of mean speed were associated with increases of speed variation, the introduction of speed limits needs to be carefully handled. Allowing more green time to the arterial traffic would decrease speed variation on the arterials but would lower the capacity of intersecting roads. The complexity of the relationships among the variables found in this study suggests that evaluation of tradeoffs between design decisions and operational conditions is critical during whole process (including planning, design and management) of building an urban arterial.

\section{ACKNOWLEDGEMENTS}

This study was jointly sponsored by the Chinese National Science Foundation (51522810, 51138003), the Science and Technology Commission of Shanghai Municipality (15DZ1204800), Jiangsu Province Collaborative Innovation Center of Modern Urban Traffic Technologies. The authors would like to thank Shanghai YOOTU Cooperation for providing the Floating Car Data.

\section{REFERENCES}

Aarts, L., Schagen, I., 2005. Driving speed and the risk of road crashes: a review. Accident Analysis and Prevention 38(2), 215-224.

Bayes, T., Price, R., 1763. An essay towards solving a problem in the doctrine of chances. Philosophical Transactions of the Royal Society 53, 370-418.

Chang, L. Y., Wang, H. W., 2006. Analysis of traffic injury severity: an application of nonparametric classification tree techniques. Accident Analysis and Prevention 38(5), 1019-1027.

Chen, M., Chien, S., 2000. Determining the number of probe vehicles for freeway travel time estimation using microscopic simulation. Proceedings of the Transportation Research Board $79^{\text {th }}$ Annual Meeting, Washington, D.C.

Cheu, R., Xie, C., Lee, D., 2002. Probe vehicle population and sample size for arterial speed estimation. Computer-Aided Civil and Infrastructure Engineering 17(1), 53-60.

Cirillo, J.A., 1968. Interstate system crash research; study II, interim report II. Public Roads 35(3), 71-76.

Cody, R. P., Smith, J. K., 2011. Applied Statistics and the SAS Programming Language. Post \& Telecom Press.

Cruzado, I., Donnell, E., 2010. Factors affecting driver speed choice along two-lane rural highway transition zones. Journal of Transportation Engineering 136(8), 755-764.

Dinh, D., Kubota, H., 2012. Profile-speed data-based models to estimate operating speeds for urban residential streets with a $30 \mathrm{~km} / \mathrm{h}$ speed limit. IATSS Research 36, 115-122.

Dixon, K., Wang, J., 2003. Evaluating operating speeds for low-speed urban streets. Proceedings of the Transportation Research Board 83 ${ }^{\text {th }}$ Annual Meeting, Washington, D.C.

Elmitiny, N., Yan, X., Radwan, E., Russo, C., Nashar, D., 2010. Classification analysis of driver's stop/go decision and red-light running violation. Accident Analysis and Prevention 42, 101111. 
Figueroa, A., Tarko, A., 2005. Speed factors on two-lane rural highways in free-flow conditions. Proceedings of the Transportation Research Board 85 ${ }^{\text {th }}$ Annual Meeting, Washington, D.C.

Gelman, A., Carlin, J. B., Stern, H. S., 2003. Bayesian data analysis. Chapman\& Hall, New York.

Gelman, A., Hill, J., 2007. Data analysis using regression and multilevel/hierarchical models. Cambridge University Press, Cambridge.

IBM Corp., 2010. IBM SPSS Statistics for Windows, Version 19.0. Armonk, NY: IBM Corp. Imperial College \& MRC, 1996-2003. WinBUGS.

Karlaftis M. G., Tarko A. P., 1998. Heterogeneity considerations in accident modeling. Accident Analysis and Prevention, 30(4), 425-433.

Liu, B., Liu, K., Mizuta, H., 2009. Study on impact of taxi status to probe car system performance. In Intelligent Transport Systems Telecommunications (ITST), 9th International Conference on IEEE, 136-137.

Park, Y., Frank F., 2006. Evaluating speed consistency between successive elements of a two-lane rural highway. Transportation Research Part A: Policy and Practice 40(5), 375-385.

Pascale, A., Deflorio, F., Nicoli, M., Chiara, B. D., Pedroli, M., 2015. Motorway speed pattern identification from floating vehicle data for freight applications. Transportation Research Part C 51, 104-119.

Poe, C., Tarris, J., Mason, J., 1998. Operating speed approach to geometric design of low-speed urban streets. Proceedings of the Transportation Research Board $78^{\text {th }}$ Annual Meeting, Washington, D.C.

SAS Institute Inc., 2002. SAS System for Windows (Version 9.3).

Seo, T., Kusakabe, T., 2015. Probe vehicle-based traffic state estimation method with spacing information and conservation law. Transportation Research Part C 59, 391-403.

Solomon, D., 1964. Crashes on main rural highways related to speed, driver and vehicle. In: Bureau of Public Roads. U.S. Department of Commerce. United States Government Printing Office, Washington, D.C.

Spiegelhalter, D., Best, N., Carlin, B., Linde, V., 2003. Bayesian measures of model complexity and fit. Journal of the Royal Statistical Society, Series B, 64, 583-616.

Verhoef E. T., Rouwendal J., Rietveld P., 1999. Congestion caused by speed differences. Journal of Urban Economics 45(3), 533-556.

Xie, K., Wang X., Huang H., Chen X., 2013. Corridor-level Signalized Intersection Safety Analysis in Shanghai, China using Bayesian Hierarchical Models. Accident Analysis and Prevention 50, 25-33.

Wang, X., Liu, H., Yu, R., Deng, B., Chen, X., Wu, B., 2014. Exploring operating speeds on urban arterials using floating car data: case study in Shanghai. Journal of Transportation Engineering, $140(9)$.

Wang, X., Fan, T., Chen, M., Deng, B., Wu, B., Tremont, P., 2015. Safety modeling of urban arterials in Shanghai, China. Accident Analysis and Prevention, 83, 57-66. 


\section{List of Tables}

Table 1 Summary of the selected arterials

Table 2 Descriptive statistics of obtained variables

Table 3 Mean speed and speed variation distributions of eight arterials

Table 4 Variable categories defined by classification tree model

Table 5 Comparison of goodness of fit among different models

Table 6 Speed variation models for each time period 
Table 1 Summary of the selected arterials

\begin{tabular}{c|c|c|c|c|c|c}
\hline $\begin{array}{c}\text { No. of } \\
\text { arterials }\end{array}$ & Direction & $\begin{array}{c}\text { Arterial } \\
\text { length }(\mathbf{m})\end{array}$ & $\begin{array}{c}\text { Minimum } \\
\text { length }(\mathbf{m})\end{array}$ & $\begin{array}{c}\text { Maximum } \\
\text { length }(\mathbf{m})\end{array}$ & $\begin{array}{c}\text { Segment } \\
\text { counts }\end{array}$ & $\begin{array}{c}\text { Average segment } \\
\text { length (m) }\end{array}$ \\
\hline \multirow{2}{*}{1} & W-E & 4601 & 175.3 & 829.0 & 11 & 418.3 \\
& E-W & 4820 & 175.3 & 829.0 & 12 & 401.7 \\
2 & W-E & 3635 & 129.3 & 395.7 & 15 & 242.3 \\
& E-W & 3976 & 129.3 & 435.8 & 16 & 248.5 \\
3 & W-E & 5085 & 152.8 & 623.4 & 15 & 339.0 \\
& E-W & 4768 & 139.0 & 598.9 & 15 & 317.9 \\
4 & W-E & 8982 & 98.2 & 629.5 & 27 & 332.7 \\
& E-W & 8518 & 74.2 & 629.5 & 26 & 327.6 \\
5 & N-S & 2353 & 79.8 & 593.6 & 9 & 261.4 \\
& S-N & 2562 & 79.8 & 593.6 & 10 & 256.2 \\
6 & N-S & 5197 & 149.6 & 861.7 & 14 & 371.2 \\
& S-N & 4536 & 139.6 & 861.7 & 13 & 348.9 \\
& W-E & 4993 & 150.6 & 573.6 & 17 & 293.7 \\
& E-W & 4820 & 150.6 & 573.6 & 16 & 301.3 \\
& W-E & 4271 & 169.0 & 797.4 & 9 & 474.5 \\
& E-W & 3473 & 169.0 & 743.7 & 9 & 385.9 \\
\hline
\end{tabular}

Note: N-S represents north to south; S-N, south to north; E-W west to east; W-E, west to east. 
Table 2 Descriptive statistics of obtained variables

\begin{tabular}{|c|c|c|}
\hline Variables & Attributes & Summary statistics \\
\hline \multirow{4}{*}{$\begin{array}{l}\text { Average signal } \\
\text { spacing } \\
\text { Signal spacing } \\
\text { variation }\end{array}$} & Arterial Level & \\
\hline & Average segment length of an arterial (m) & Mean $=332.56 ;$ SD $=66.21$ \\
\hline & Standard deviation of segment length of an arterial (m) & Mean $=160.58 ;$ SD $=42.22$ \\
\hline & Segment Level & \\
\hline \multicolumn{3}{|c|}{ Geometric design features } \\
\hline Segment length & $\begin{array}{l}\text { Length of segment using signalized intersection as } \\
\text { segment boundary }(\mathrm{m})\end{array}$ & Mean $=327.30 ;$ SD $=165.70$ \\
\hline Number of lanes & $\begin{array}{l}\text { Total number of lanes for segments, ranging from two } \\
\text { to six }\end{array}$ & $\begin{array}{l}\text { Two lanes: } 24 \\
\text { Three lanes: } 77 \\
\text { Four lanes: } 101 \\
\text { More than five lanes: } 32\end{array}$ \\
\hline Median & $\begin{array}{l}1 \text { for with median } \\
0 \text { for no median }\end{array}$ & $\begin{array}{l}\text { With median:202 } \\
\text { No median:32 }\end{array}$ \\
\hline $\begin{array}{l}\text { Separation between } \\
\text { motor and bicycle } \\
\text { lanes }\end{array}$ & $\begin{array}{l}1 \text { for with separation } \\
0 \text { for no separation }\end{array}$ & $\begin{array}{l}\text { With separation: } 180 \\
\text { No separation: } 54\end{array}$ \\
\hline Access & Total number of access points along segments & Mean:1.88; SD: 0.88 \\
\hline Bus stops & $\begin{array}{l}1 \text { for with bus stops } \\
0 \text { for no bus stops }\end{array}$ & $\begin{array}{l}\text { With bus stops: } 112 \\
\text { Without bus stops: } 122\end{array}$ \\
\hline Bus rapid transit & $\begin{array}{l}1 \text { for with bus rapid transit } \\
0 \text { for no bus rapid transit }\end{array}$ & $\begin{array}{l}\text { With bus rapid transit: } 96 \\
\text { Without bus rapid transit: } 138\end{array}$ \\
\hline \multicolumn{3}{|l|}{ Traffic volume } \\
\hline $\begin{array}{l}\text { Traffic volume for } \\
\text { AM peak }\end{array}$ & $\begin{array}{l}\text { Average through lane volume along the arterials in the } \\
\text { AM peak hours (pcu/h/lane) }\end{array}$ & Mean $=458.18 ;$ SD $=139.94$ \\
\hline $\begin{array}{l}\text { Traffic volume for } \\
\text { Noon off-peak }\end{array}$ & $\begin{array}{l}\text { Average through volume per lane along the arterials in } \\
\text { the Noon off- peak hours (pcu/h/lane) }\end{array}$ & Mean $=394.76 ;$ SD $=123.05$ \\
\hline $\begin{array}{l}\text { Traffic volume for } \\
\text { PM peak }\end{array}$ & $\begin{array}{l}\text { Average through volume per lane along the arterials in } \\
\text { the PM peak hours (pcu/h/lane) }\end{array}$ & Mean $=505.53 ; \mathrm{SD}=187.65$ \\
\hline \multicolumn{3}{|c|}{ Traffic control parameters } \\
\hline Numbers of phases & Numbers of phases per cycle, ranging from two to six. & $\begin{array}{l}\text { Two phases: } 23 \\
\text { Three phases: } 127 \\
\text { Four phases: } 50 \\
\text { Five/Six phases: } 34\end{array}$ \\
\hline Cycle length & Length of cycle (s) & Mean $=181.03 ;$ SD $=31.50$ \\
\hline Green time & Green time for the through lanes along the arterial (s) & Mean $=89.24 ;$ SD $=26.97$ \\
\hline \# of observations & 234 & \\
\hline
\end{tabular}


Table 3 Mean speed and speed variation distributions of eight arterials

\begin{tabular}{cccccccc}
\hline \multirow{2}{*}{$\begin{array}{c}\text { No. of } \\
\text { arterials }\end{array}$} & \multirow{2}{*}{ Direction } & \multicolumn{3}{c}{ Mean speed $(\mathbf{k m} / \mathbf{h})$} & \multicolumn{3}{c}{ Speed variation $(\mathbf{k m} / \mathbf{h})$} \\
\cline { 3 - 7 } & & AM & Noon & PM & AM & Noon & PM \\
\hline \multirow{2}{*}{1} & W-E & $31.27(5.64)$ & $41.41(6.16)$ & $34.33(6.32)$ & $15.51(3.15)$ & $18.55(2.35)$ & $16.27(3.70)$ \\
& E-W & $40.17(7.33)$ & $43.90(7.00)$ & $35.79(7.79)$ & $18.53(4.19)$ & $18.32(4.29)$ & $16.59(3.65)$ \\
2 & W-E & $36.14(6.69)$ & $40.29(7.10)$ & $35.57(8.31)$ & $15.54(3.82)$ & $17.22(3.99)$ & $15.34(3.89)$ \\
& E-W & $36.11(8.59)$ & $40.23(7.47)$ & $32.26(9.26)$ & $16.11(3.01)$ & $16.61(2.99)$ & $14.38(3.91)$ \\
\multirow{2}{*}{3} & W-E & $33.86(7.76)$ & $39.26(6.21)$ & $34.31(8.35)$ & $15.98(3.82)$ & $17.78(3.41)$ & $15.74(3.46)$ \\
& E-W & $36.04(5.74)$ & $37.87(7.61)$ & $31.01(6.35)$ & $16.26(2.94)$ & $16.28(3.36)$ & $14.64(2.48)$ \\
\multirow{2}{*}{4} & W-E & $41.20(9.16)$ & $42.52(5.78)$ & $36.84(7.88)$ & $16.33(4.27)$ & $17.33(4.53)$ & $16.10(4.72)$ \\
& E-W & $36.97(7.89)$ & $43.66(6.94)$ & $40.12(7.13)$ & $16.41(3.82)$ & $18.11(4.52)$ & $17.24(3.77)$ \\
\multirow{2}{*}{5} & N-S & $28.98(5.92)$ & $39.98(4.22)$ & $36.30(7.50)$ & $12.94(2.18)$ & $16.14(3.56)$ & $14.97(3.76)$ \\
& S-N & $37.97(4.11)$ & $37.70(6.48)$ & $29.46(4.59)$ & $16.40(3.15)$ & $16.22(3.14)$ & $13.42(2.98)$ \\
6 & N-S & $29.49(8.41)$ & $33.09(6.10)$ & $26.63(5.77)$ & $13.09(2.67)$ & $14.92(3.56)$ & $14.38(3.19)$ \\
& S-N & $28.00(3.61)$ & $31.65(4.57)$ & $27.27(5.87)$ & $14.53(3.84)$ & $14.76(3.91)$ & $11.99(2.57)$ \\
7 & W-E & $30.32(4.73)$ & $36.73(7.48)$ & $33.48(9.08)$ & $12.85(2.68)$ & $16.51(3.93)$ & $15.43(3.33)$ \\
& E-W & $35.23(6.90)$ & $39.94(6.00)$ & $28.94(6.14)$ & $16.13(2.69)$ & $16.24(2.94)$ & $14.15(2.95)$ \\
\multirow{2}{*}{8} & W-E & $34.38(7.24)$ & $40.90(4.96)$ & $36.49(5.11)$ & $16.89(3.45)$ & $18.08(4.73)$ & $16.98(4.02)$ \\
& E-W & $35.23(6.31)$ & $37.37(5.87)$ & $28.23(9.13)$ & $16.23(3.19)$ & $16.90(2.08)$ & $12.86(3.75)$ \\
\hline
\end{tabular}

Note: Standard deviations for the coefficients are shown in parenthesis. 
Table 4 Variable categories defined by classification tree model

\begin{tabular}{l|c}
\hline \multicolumn{1}{c|}{ Variable } & Classes or categories \\
\hline Traffic volume for AM peak $(\mathrm{pcu} / \mathrm{h} / \mathrm{lane})$ & $0(<=490.8), 1(>490.8)$, \\
Traffic volume for Noon off-peak $(\mathrm{pcu} / \mathrm{h} / \mathrm{lane})$ & $0(<=457.0), 1(>457.0)$ \\
Traffic volume for PM peak $(\mathrm{pcu} / \mathrm{h} / \mathrm{lane})$ & $0(<=568.3), 1(>568.3)$ \\
Mean speed for AM peak $(\mathrm{km} / \mathrm{h})$ & $0(<=32.60), 1(>32.60$ and $<=36.70), 2(>36.70)$ \\
Mean speed for Noon off-peak $(\mathrm{km} / \mathrm{h})$ & $0(<=29.40), 1(>29.40$ and $<=39.60), 2(>39.60)$ \\
Mean speed for PM peak $(\mathrm{km} / \mathrm{h})$ & $0(<=21.80), 1(>21.80$ and $<=25.90), 2(>25.90$ and $<=33.70)$, \\
\hline
\end{tabular}


Table 5 Comparison of goodness of fit among different models

\begin{tabular}{l|c|c|c}
\hline \multicolumn{1}{c|}{ Model description } & \multicolumn{3}{c}{ DIC } \\
\cline { 2 - 4 } & AM peak & Noon off-peak & PM peak \\
\hline 1.Fixed independent variables & 1204.3 & 1270.1 & 1259.8 \\
$\begin{array}{l}\text { 2. Fixed independent variables, speed and volume as } \\
\text { categorical variables }\end{array}$ & 1207.3 & 1276.2 & 1263.3 \\
$\begin{array}{l}\text { 3.Speed and volume as random variables } \\
\text { 4.Speed as a random variable, volume as a categorical } \\
\text { variable }\end{array}$ & 1154.5 & 1236.2 & 1206.8 \\
\hline
\end{tabular}


Table 6 Speed variation models for each time period

\begin{tabular}{|c|c|c|c|c|c|c|c|c|c|c|c|c|}
\hline \multirow{2}{*}{ Influencing variable } & \multicolumn{4}{|c|}{ AM peak } & \multicolumn{4}{|c|}{ Noon off-peak } & \multicolumn{4}{|c|}{ PM peak } \\
\hline & Mean & SD & $5.00 \%$ & $95.00 \%$ & Mean & SD & $5.00 \%$ & $95.00 \%$ & Mean & SD & $5.00 \%$ & $95.00 \%$ \\
\hline $\begin{array}{c}\text { Intercept } \\
\text { (Std. in parenthesis) }\end{array}$ & $\begin{array}{c}10.75 \\
(1.275)\end{array}$ & $\begin{array}{l}1.308 \\
(0.576)\end{array}$ & 8.652 & 12.94 & $\begin{array}{c}15.72 \\
(1.201)\end{array}$ & $\begin{array}{c}1.636 \\
(0.495)\end{array}$ & 13.06 & 18.41 & $\begin{array}{c}7.796 \\
(0.908)\end{array}$ & $\begin{array}{c}1.1 \\
(0.408)\end{array}$ & 6.009 & 9.592 \\
\hline Number of lanes(5) & 1.691 & 0.724 & 0.492 & 2.883 & 1.874 & 0.805 & 0.548 & 3.202 & 1.189 & 0.679 & 0.066 & 2.306 \\
\hline Number of lanes(4) & 1.471 & 0.54 & 0.59 & 2.35 & 2.255 & 0.607 & 1.246 & 3.247 & 1.289 & 0.507 & 0.457 & 2.122 \\
\hline Number of lanes(2) & 0.407 & 0.775 & -0.859 & 1.676 & 0.014 & 0.854 & -1.377 & 1.416 & -0.143 & 0.725 & -1.333 & 1.061 \\
\hline Number of lanes ( 3 , base case) & - & - & - & - & - & - & - & - & - & - & - & - \\
\hline Access & 0.737 & 0.423 & 0.05 & 1.431 & 1.092 & 0.481 & 0.295 & 1.879 & 1.202 & 0.401 & 0.546 & 1.86 \\
\hline Bus stops (Base: no bus stops) & 0.784 & 0.408 & 0.112 & 1.452 & 0.575 & 0.477 & -0.208 & 1.358 & 0.798 & 0.392 & 0.155 & 1.448 \\
\hline Traffic volume & -1.086 & 0.462 & -1.84 & -0.323 & -0.765 & 0.56 & -1.689 & 0.158 & -1.679 & 0.457 & -2.435 & -0.94 \\
\hline $\begin{array}{c}\text { Mean speed } \\
\text { (Std. in parenthesis) }\end{array}$ & $\begin{array}{c}0.186 \\
(0.039)\end{array}$ & $\begin{array}{l}0.033 \\
(0.02)\end{array}$ & 0.132 & 0.24 & $\begin{array}{l}0.075 \\
(0.02)\end{array}$ & $\begin{array}{c}0.04 \\
(0.013)\end{array}$ & 0.003 & 0.139 & $\begin{array}{c}0.24 \\
(0.026)\end{array}$ & $\begin{array}{c}0.027 \\
(0.011)\end{array}$ & 0.195 & 0.283 \\
\hline Green time & -0.03 & 0.009 & -0.045 & -0.016 & -0.039 & 0.01 & -0.056 & -0.023 & -0.019 & 0.008 & -0.033 & -0.006 \\
\hline \# of Observations & \multicolumn{4}{|c|}{234} & \multicolumn{4}{|c|}{234} & \multicolumn{4}{|c|}{234} \\
\hline
\end{tabular}




\section{List of Figures}

Figure 1 Speed profile along an arterial (one vehicle sample)

Figure 2 Speed profiles in segments along two selected arterials during AM peak hours Figure 3 Distributions of speed variation on Arterial 5 during different time periods

Figure 4 Mean speeds and speed variations across all segments during each time period 

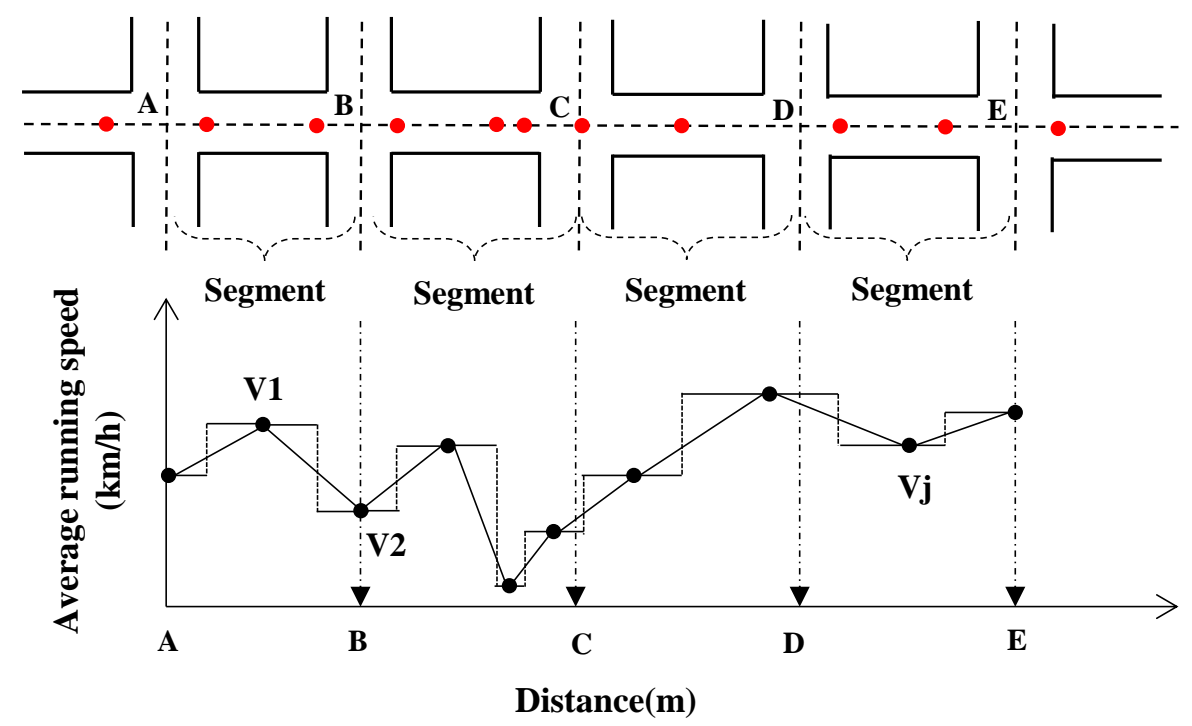

Figure 1 Speed profile along an arterial (one vehicle sample). 

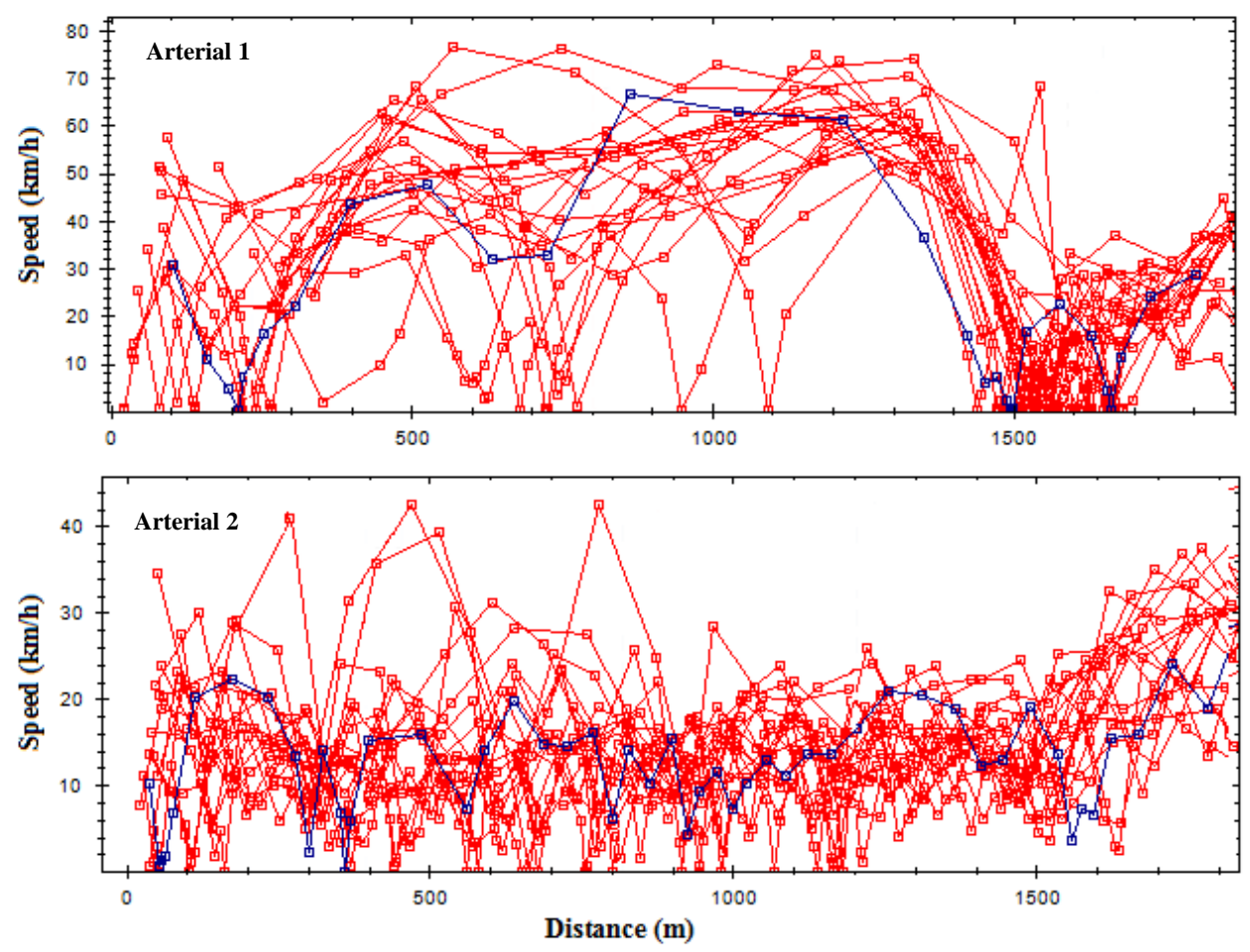

Legend $\square-$ Speed profile for one taxi $\quad \square \quad$ Speed profile for other taxis

Figure 2 Speed profiles in segments along two selected arterials during AM peak hours 


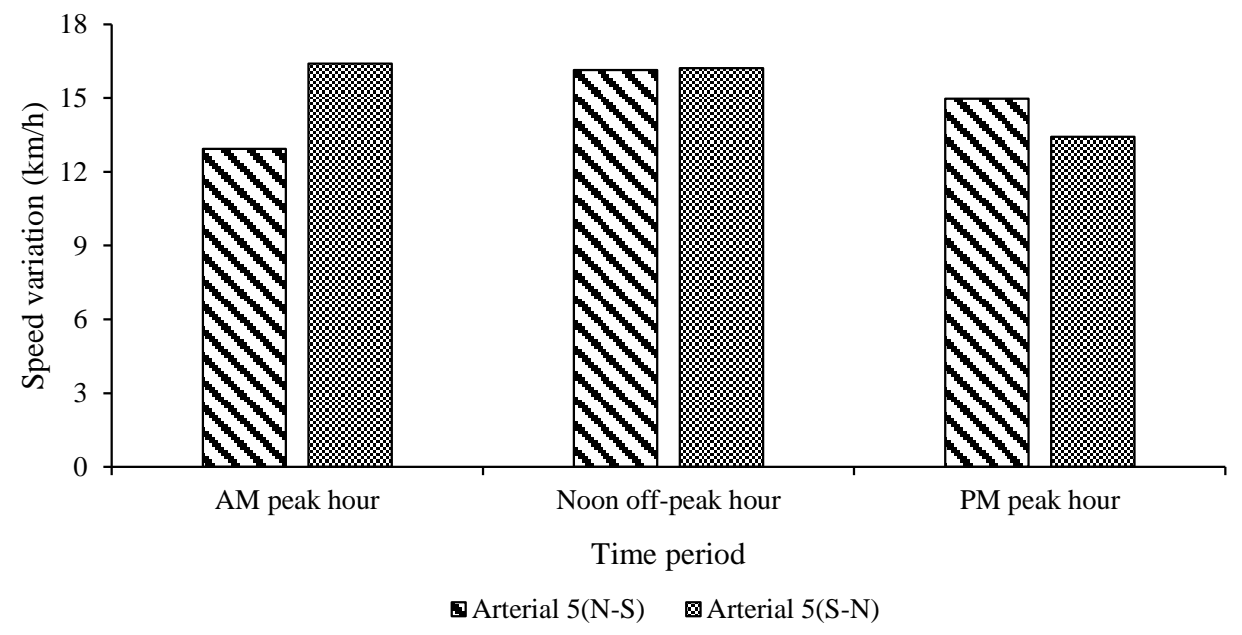

Figure 3 Distributions of speed variation on Arterial 5 during different time periods 


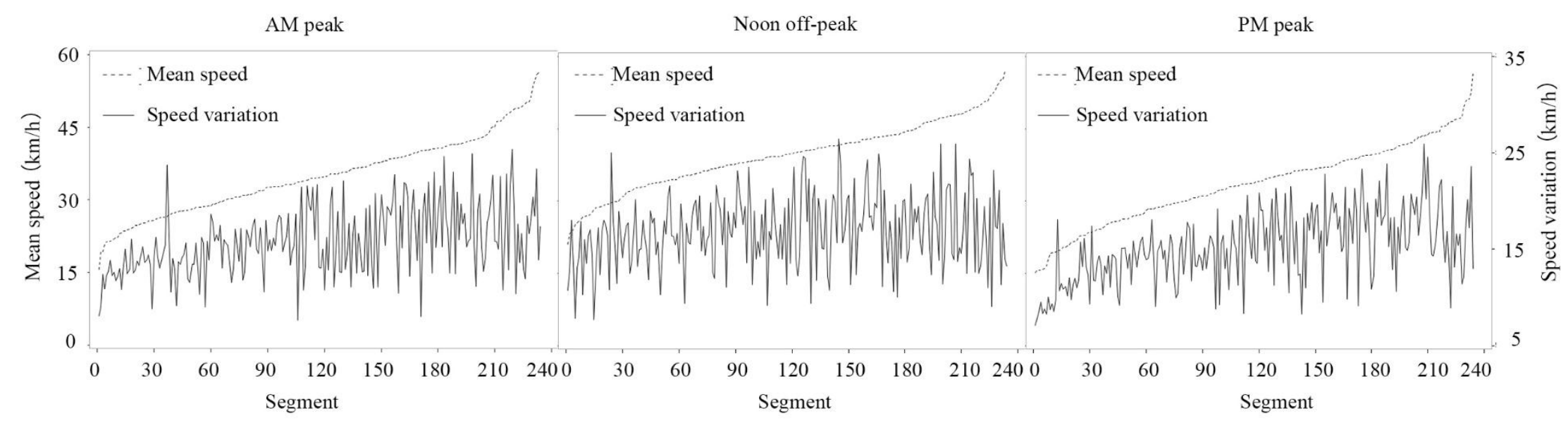

Figure 4 Mean speeds and speed variations across all segments during each time period 